\title{
SURFACE-MICROMACHINED BEAMS WITHOUT SPRING EFFECT OF ANCHOR STEP-UP
}

\author{
Lan V. Ngo, Phyllis Nelson*, and Chang-Jin Kim \\ Mechanical and Aerospace Engineering Department \\ *Electrical Engineering Deparment \\ University of California, Los Angeles \\ Los Angeles, CA 90095
}

\begin{abstract}
A new anchor design for a surface-micromachined beam is proposed for eliminating the extra spring effect at the step-up anchor. With a minor modification in the sacrificial-layer mask layout, the spring effect is eliminated by forming a reinforcement hump at the beam anchor. The design idea is verified by comparing the resonant frequencies of the conventional and proposed microbeams numerically in FEM analysis and experimentally with a Michaelson laser interferometer.
\end{abstract}

\section{INTRODUCTION}

Surface-micromachined beams are a main component in microelectromechanical systems (MEMS). When surfacemicromachined beam is fabricated, it has a step-up (Fig. 1a) at the anchor, which contributes an extra spring effect. For many MEMS applications, the anchor spring effect is not accounted for in the design and analysis, leaving questions in the accuracy of their performance. Although this effect can be accounted for with additional beam theory equations, they often lead to a complex analysis. For simplicity, ideal fixed-end boundary condition (Fig. 1c) is commonly used for modeling the boundary condition of the surface-micromachined beams. However, results showed that care must be taken when surface-micromachined beams are modeled as a cantilever beam [1].

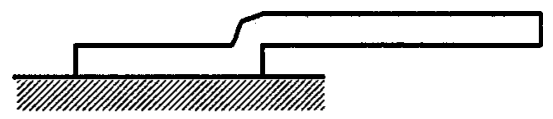

a. Typical surface-micromachined beam with step-up.

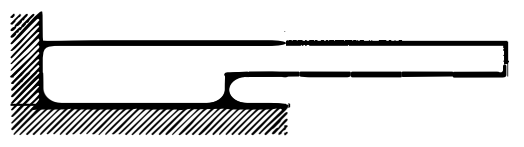

b. Limiting case.

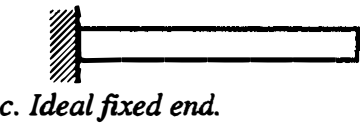

Fig. 1: Various Anchor Types

Work have been done to fabricate a surface-micromachined beam similar to a cantilever beam of the limiting case and to alleviate this problem, so that analytical results are more accurate. However, the design usually requires modifying the process and/or introducing new process steps [2]. In this work, a surfacemicromachined beam is designed to eliminate the step-up spring effect. Without altering the fabrication process or adding new processing steps, it makes only a simple modification in the layout of the sacrificial layer. The idea of the mask modification is to form a reinforcement at the step-up anchor by taking advantage of the gap-filling characteristic of LPCVD, so that the spring effect is eliminated and the beam can be modeled more accurately as a simple cantilever beam. Since the anchor condition of surfacemicromachined beam has a limit, the goal of this project is to design a micro-beam that has an anchor condition approaching the limit (Fig. 1b). The design idea is initially verified by FEM analysis, and microbeams are made and tested.

\section{DESIGN AND FABRICATION}

Fig. 2 illustrates two surface-micromachined beams with different anchors: typical surface-micromachined beam with conventional anchor (Fig. 2a) and the microbeam with the new purposed anchor (Fig 2b). As show in the figure, both microbeams have the same fabrication process; the only difference is the additional PSG block made by minor modification on the sacrificial layer mask pattern. When conventional surfacemicromachined beam is fabricated, all the sacrificial layer (usually PSG) is removed in the anchoring region, Fig. 2a. However, when the beam with new reinforced anchor is made, a block of PSG is left in the anchoring region to create a narrow gap, Fig. $2 b$. If the gap is narrow enough, the subsequent polysilicon layer will fill the gap, forming a reinforced anchor and approaching the limit condition of Fig. lb.

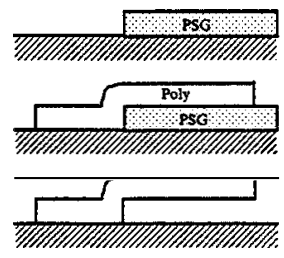

(a)

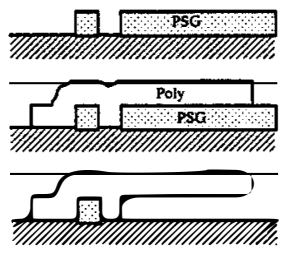

(b)
'Fig. 2: (a) Microbeam with conventional anchor. (b) Microbeam with proposed anchor

Fig. 3 is a demonstration of the polysilicon filling the narrow oxide gap. It is fabricated by MCNC/MUMPs processing foundry [3]. The polysilicon and the oxide layers are $2 \mu \mathrm{m}$ thick, and the gaps are $3 \mu \mathrm{m}$ and $2 \mu \mathrm{m}$ in the mask level. The fabricated size of the gaps are about $0.5 \mu \mathrm{m}$ wider because of the overetching during the process. The picture shows that the new design technique may make beams approaching the limit case (Fig. 1b).

The testing beams are also fabricated by using the standard MUMPs foundry. Since the fundamental frequency of the beam is measured by using laser interferometer, the beam is made relatively wide $(50 \mu \mathrm{m})$. However, the wide beam is subject to squeeze film damping effect which may attenuate the vibration amplitudes of the beam and decrease the sensing signals. In an 
attempt to minimize this effect, a post process is performed to remove the substrate underneath the beam.

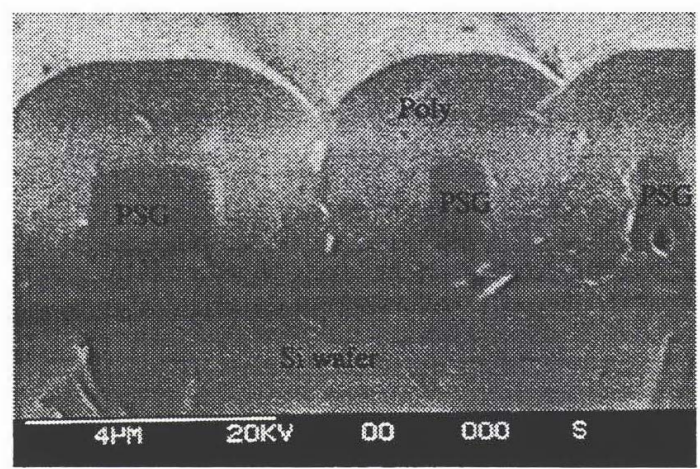

Fig. 3: Polysilicon filling the oxide gaps. The gaps are $3 \mu \mathrm{m}$ (left) and $2 \mu \mathrm{m}$ (right) width. The poly and the PSG are $2 \mu \mathrm{m}$ thick.

This post process is a non-mask etching step by taking advantage of the overetching during the MUMPs fabrication. The overetch will define a poly protected mask by opening pit on the substrate while the oxide still covers the poly. Fig. 4 is the process flow of the post etch. After removing the protective photoresist in the MUMPs dice, xenon diflouride $\left(\mathrm{XeF}_{2}\right)$ is used to remove the silicon substrate underneath the beams. The nitride layer underneath the beam is etched by phosphoric acid $\left(\mathrm{H}_{3} \mathrm{PO}_{4}\right)$. Finally, the structure is released with HF.

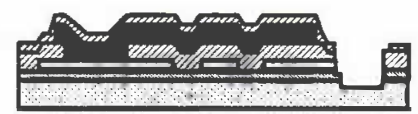

Structure after MUMPs process.

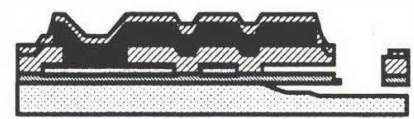

Structure after XeF 2 etch.

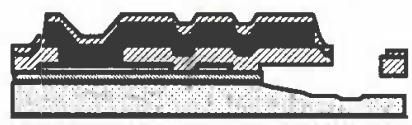

Structure after H3PO4 etch.

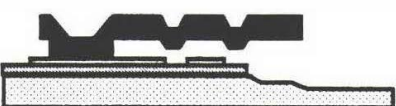

Structure after released.

Fig. 4: Process flow for the post etch. Poly2 and metal layers of MUMPs are not shown in the figure.

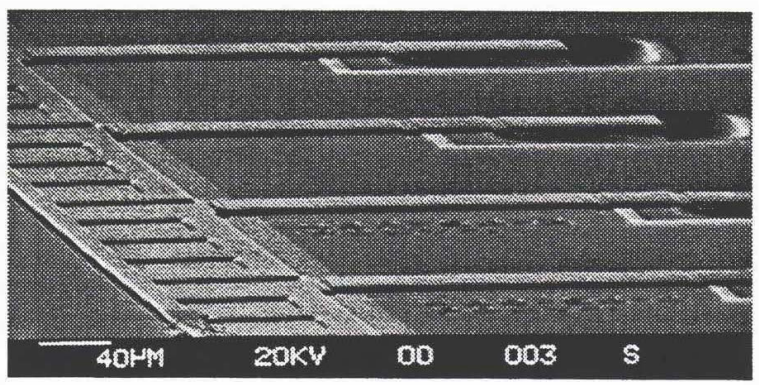

Fig. 5: Microbeams with conventional anchor and proposed anchors. Substrate underneath the beam ends are recessed.
Fig. 5 is the complete beams after the post processing. Beams with conventional anchor and identical beams with proposed anchor are placed next to each other as pairs for direct comparison. Fig. 6 shows the proposed anchor at the base of a microbeam.

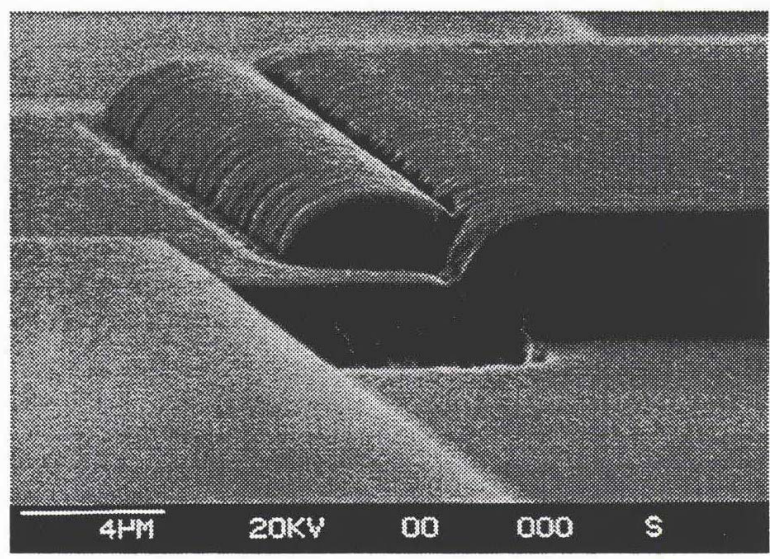

Fig. 6: Proposed anchor at the base of a microbeam.

\section{FINITE ELEMENT MODELING}

FEM models are developed and coded in a commercial PATRAN package for predicting the resonant frequency. The beam is modeled as a two-dimensional problem. Beams with three different types of anchoring are analyzed and compared. Fig 7 shows the models of the beams with different types of anchors: conventional step-up anchor, proposed reinforced anchor, and limiting case. For the step-up anchor and the proposed reinforced anchor, the curve profile of the anchor is fed directly from the actual structure fabricated, i.e., from the SEM pictures of the cross section of the microstructures.

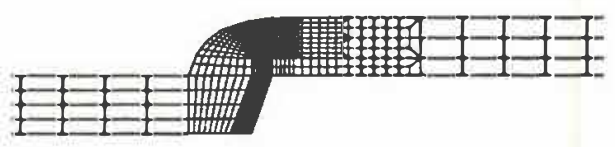

(a) Conventional anchor.

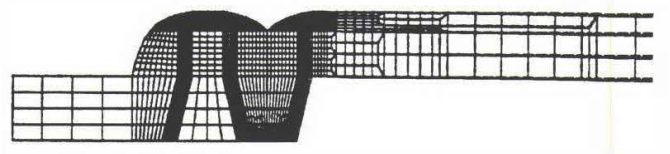

(b) Proposed anchor.

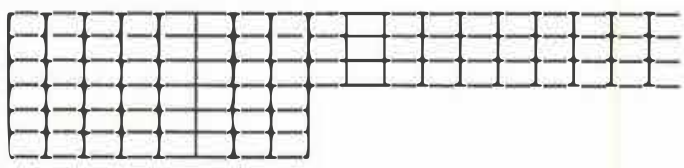

(c) Limiting case.

Fig. 7: FEM models of beams with various anchors. Curved profiles in (a) and (b) are based on the actual cross-section of the fabricated structures. 


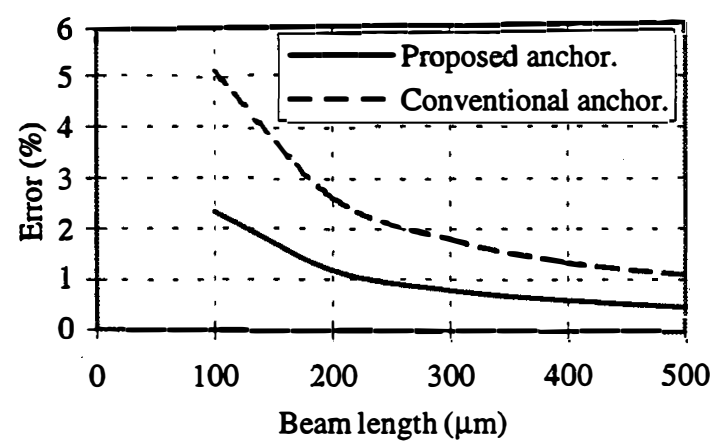

Fig. 8: Deviation of the resonant frequency of microbeams with two different anchors from the case of fixed end beams.

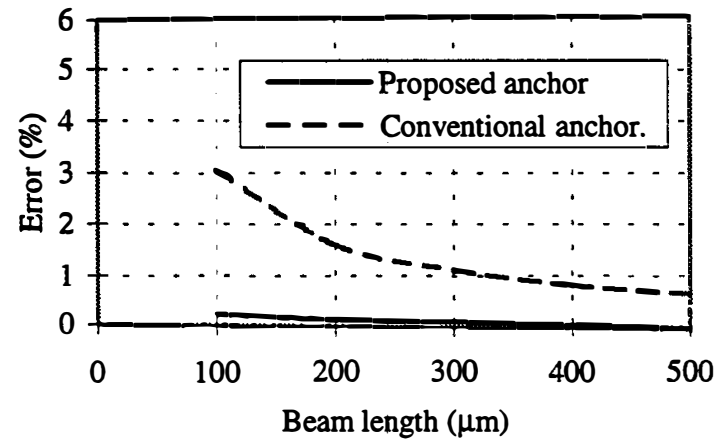

Fig. 9: Deviation of the resonant frequency of microbeams with two different anchors from that of the limiting case.

By analyzing the resonant frequency of beams with various anchors, the spring effect of different anchors are evaluated. Fig. 8 compares the percentage deviation of the resonant frequency of microbeam with conventional and proposed anchor from the case of an ideal fixed end support (Fig. . 1c). The deviation is the error in predicting resonant frequency of the beam if an ideal fixed end is assumed for analysis. The curve for the proposed anchor almost coincides with that for the limiting case (Fig. 1b) as evidenced by Fig. 9, which compares the errors of the conventional anchor and proposed anchor when they are approximated as the limiting case. The proposed anchor has negligible error when approximated as the limiting case support. Same comparison has been made for microbridges in Fig. 10 and Fig. 11, as opposed to the cantilevers.

\section{MEASUREMENT}

To evaluate the anchor design, the motion of microbeams with the conventional anchor and the proposed is monitored by a Michaelson interferometer. Fig. 12 is a schematic drawing of the apparatus. Mirrors $\mathrm{M} 1$ and M2 are used to align a helium neon $(\mathrm{HeNe})$ laser used as the light source for the interferometer. This input beam is divided into two orthogonal beams by a beam splitter (BS). One beam (B1), which is used as the reference, is reflected back to the beam splitter by a mirror (M3) mounted on a piezoelectric translator. The other beam leaving the beam splitter(B2) is focused on the microstructure by a lens. The reflection from the device under test retums through the lens to the beam splitter, where it is combined with the reference beam. Upon their retum to the beam splitter, the transmitted portion of B1 and the reflected portion of B2 pass through a

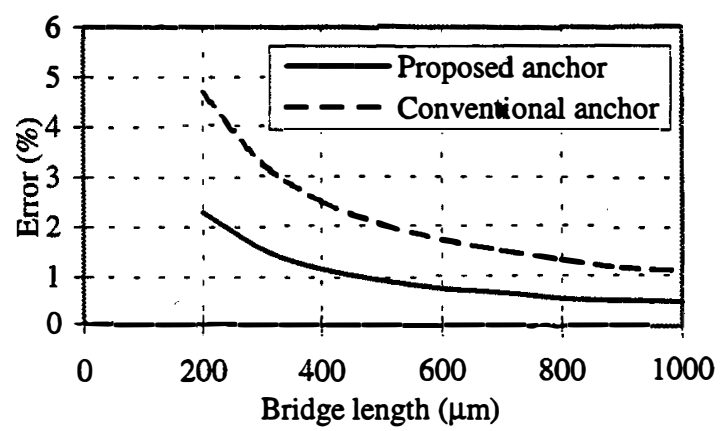

Fig. 10: Deviation of the resonant frequency of microbridges with two different anchors from the case of fixed end bridges.

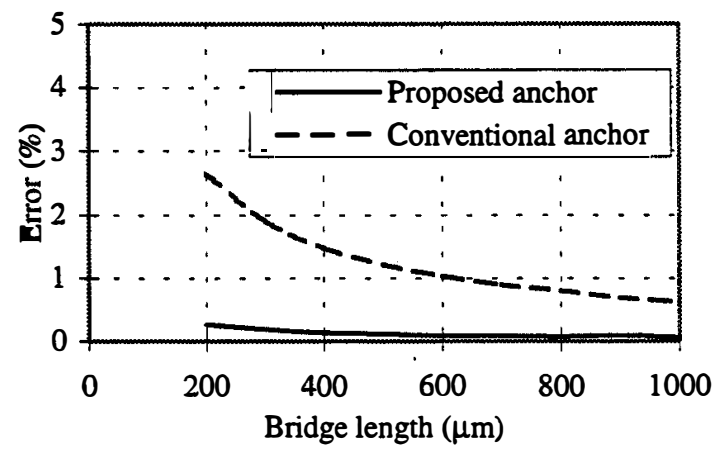

Fig. 11: Deviation of the resonant frequency of microbridges with two different anchors from the case of the limiting case.

diverging lens. The system is focused to give a projected image of the device under test at the plane of an aperture (A) with beam B2 alone. When the retum reflection of B1, the reference, is added, an interference pattem is superimposed on the image due to the difference in the phase of the two beams.

The intensity of the light passing the aperture is detected by a silicon photodiode having a time constant of less than $1 \mu \mathrm{s}$. By adjusting the linear translator, the relative optical path lengths traveled by B1 and B2 can be adjusted for a linear small signal response to displacement of the device under test. A lock-in amplifier is used to detect the device motion by isolating the intensity variations at the appropriate frequency. Measurement of the fringe contrast allows small signal intensity changes to be converted to absolute displacement of the test structure. Therefore, the dynamics response of the system can be evaluated.

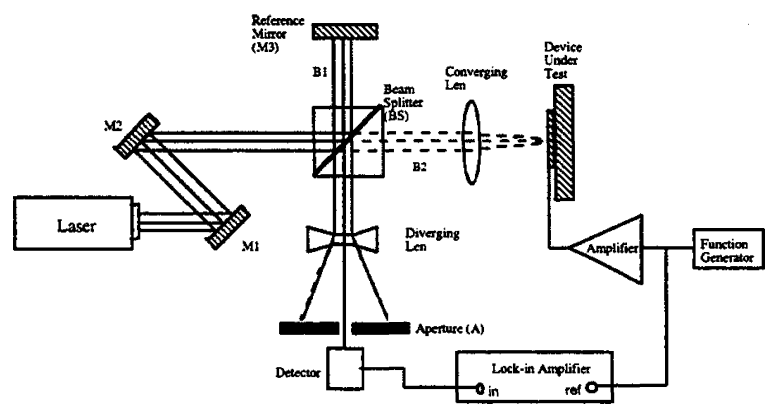

Fig. 12: The Michaelson interferometer used to measure the resonant frequency. 
The microstructures are excited by an electrostatic actuator, which is built in the test structure. At a constant DC offset, sinusoidal signals are applied to the system and swept at a frequency range to determine the resonance.

We estimated the resonant frequency of $500 \mu \mathrm{m}$ long, $50 \mu \mathrm{m}$ wide, $2 \mu \mathrm{m}$ thick microbeams with both types of anchors from the measured phase shifts of their dynamic responses. Fitting the phase data for the conventional microbeam to that expected for a second order damped spring mass system yields a resonant frequency of $8.92 \mathrm{kHz}$. The data points and fit are shown in Fig. 13. (This fit also includes a phase offset of -28 degrees to account for the phase response of the actuation and measurement electronics.) The measured phase data for the structure with the proposed anchor, which is also plotted in Fig. 13, cannot be fit to the simple second order form. However, this structure yields a smaller phase shift at any given frequency than the conventional one, which is consistent with the prediction that the proposed structure is closer to the fixed end condition (i.e., stiffer).

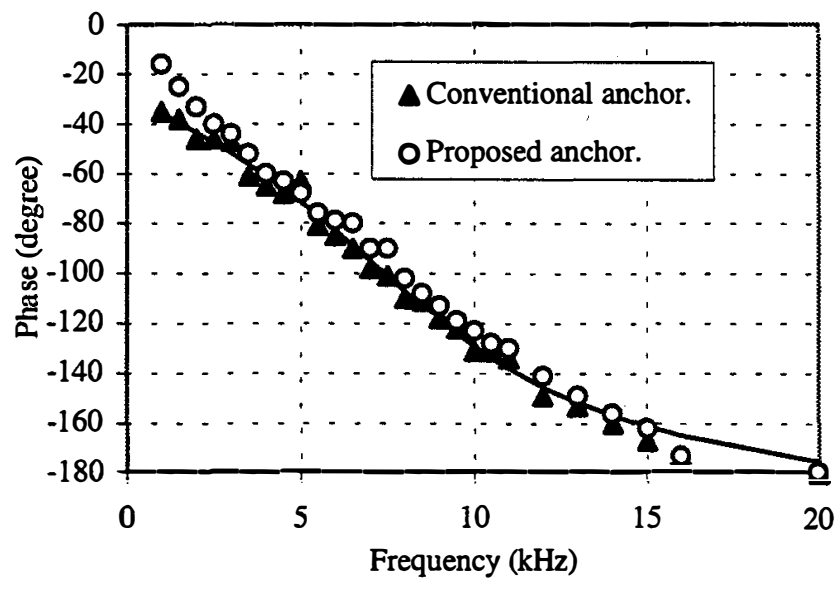

Fig. 13: Phase response of microbeams with conventional anchor and proposed anchor. (The fit of the conventional anchor to a second order damped spring mass system is shown as a solid line.)

Our measurements are limited to less than $20 \mathrm{kHz}$ by the bandwidth and slew rate of the amplifier used to actuate the microbeams. Thus, we have only examined relatively long microbeams with low frequency resonance. However, these are structures in which the effect of the anchor is relatively small. We are currently improving the actuation system, and will then study shorter beams with a larger dependence on the anchor structure. In addition, we will investigate the possibility of using an acoustooptic modulator and optical heterodyne detection to improve the signal-to-noise ratio of the measurement system.

\section{CONCLUSION}

A new anchor that reduces the step up spring effect in surface-micromachined beams has been designed and fabricated. This new design requires no change in the existing fabrication process. The modified anchor performance has been verified by FEM analysis and measured experimentally. The results show that the anchor spring can be eliminated by minor modification in the sacrificial layer layout.

\section{ACKNOWLEGEMENT}

The authors would like to thank Prof. Oscar Stafsudd for valuable discussion and the loan of experimental equipment and Prof. Yang for the use of CAD lab.

\section{REFERENCES}

1. Q. Meng, M. Mehregany, and R. L. Mullen, "Theoretical Modeling of Microfabricated Beams Elastically Restrained Supports," J. Microelectromechanical Systems, Vol. 2, (1993), pp. 128-137.

2. Y.X. Li, P.J. French, and R.F. Wolffenbuttel, "Plasma Planarization for Sensor Applications," $J$. Microelectromechanical Systems, Vol. 4, (1995), pp. 32-138.

3. Multi-User MEMS Process (MUMPs) of the MCNC Center for Microelectronics Systems Technologies, Research Triangle Park, NC 27709-2889.

4. F. I. Chang, R. Yeh, G. Lin, P. B. Chu, E. Hoffman, E. J. J. Kruglick, K. S. J. Pister, and M. H. Hecht, "Gas-Phase Silicon Micromachining With Xenon Difluoride," SPIE 1995 Symposium on Micromachining and Microfabrication, Austin, Texas, (1995).

5. P. B. Chu, P. R. Nelson, M. L. Tachiki, and K. S. J. Pister, "Dynamics of Polysilicon Parallel-Plate Electrostatic Actuators," Proc. 8th Int. Conf. on Solid-State Sensor and Actuator, Stockholm, Sweden, (1995), pp. 356-359. 